\title{
A Novel Synthesis of 2-Aryl-4-quinolones from 2-Aminobenzoic Acids
}

\author{
Jae In Lee* and Jung Suk Youn \\ Deparment of Chemistry and Plant Resources Research Institute. College of Natural Science, \\ Duksung Women's Chiversin, Seoul 132-714, Korea. "E-mail: jileéaduksung ack
}

Received Julv 1. 2008

Key Words : 2-Aryl-4-quinolones. Cyclization. Condensation. Dehydrogenation

\begin{abstract}
2-Aryl-4-quinolones aza analogs of flavones. have played a central role in medicinal chemistry because they possess potent antimitotic antitumor effects through inhibition of tublin polymerization at the colchicine site. The general method for preparing 2-aryl-4-quinolones is a condensation of anilines with ethyl benzoylacetates. ${ }^{2}$ thioalkylidene-1.3dioxane-4.6-diones ${ }^{3}$ and diethyl 2-(ethoxymethylene)malonate ${ }^{t}$ in diphenyl ether at $240-250^{\circ} \mathrm{C}$. This process is amenable for scale-up and the starting material anilines are widely available. but it can lead to regioisomers depending on the structure of anilines. The cyclization of $N$-(2-acetylphenyl)benzamides. which are prepared from the benzoylation of 2'-aminoacetophenones with benzoyl chlorides ${ }^{5}$ or Friedel-Crafts acylation of $N$-phenylbenzanides with acetyl chloride. "with potassium $t$-butoxide gives 2 -aryl-4-quinolones. However. Friedel-Crafts acylation gives $N$-(2-acetylphenyl)benzamides in moderate yields together with its regioisomers. LDA-mediated cyclization of ketimines which are prepared by the condensation of anthranilides with acetophenones also gives 2-aryl-4-quinolones. but it fails when electron-withdrawing groups are present on the 2phenyl ring. ${ }^{7}$ The reaction of 2.3-dihydroquinolin- $4(1 H)$ ones. which are prepared by the cyclization of 2 -amino-
\end{abstract} chalcones with polyphosphoric acid and micro-assisted K10 clay $^{9}$ or $\mathrm{InCl}_{3}-\mathrm{SiO}_{2}{ }^{10}$ with thallium $p$-toly lsulphonate ${ }^{1]}$ results in dehydrogenation to give 2-aryl-4-quinolones. Altematively, the reaction of succinimidyl esters derived from 2-aminobenzoic acids with anions of $\beta$-keto esters affords 2-aryl-4-quinolones via $\beta$-anilinoketo esters but an excess of $\beta$-keto esters is used and yields are low. ${ }^{12}$

However there are few reports on the preparation of 2 aryl-4-quinolones from 2 -aminoacetophenones. The use of 2 -aminoacetophenones for preparing 2-aryl-4-quinolones can avoid the undesirable reaction during the acetylation of $N$-phenylbenzamides. As part of our extending studies of flavonoids. ${ }^{13}$ we report that 2-aryl-4-quinolones can be synthesized via 2'-aminoacetophenones derived newly from 2-aminobenzoic acids.

The preparation of $2^{\prime}$-aminoacetophenone was attempted by treating 2-aminobenzoic acid with 3 equiv of methyllithium in THF. $\mathrm{Et}_{2} \mathrm{O}$, and DME varying solvent at $0{ }^{\circ} \mathrm{C}$. Under these conditions 2'-aminoacetophenone was obtained in $65 \%, 57 \%$, and $80 \%$ yield. respectively. after $3 \mathrm{~h}, 2 \mathrm{~h}$. and $1 \mathrm{~h}$. respectively. Thus the preparation of 2 -aminoacetophenones 2 was carried out by the slow addition of 3 equiv of methyllithium to a solution of 2-aminobenzoic acids 1 in $\mathrm{DME}$ at $0^{\circ} \mathrm{C}$ (Scheme 1). After being stirred for $1 \mathrm{~h}$. the resulting tan solution containing white precipitate was separated by usual acidic workup and the condensed residue was subjected to silica gel chromatography using $30 \%$ $\mathrm{EtOAc} / n$-hexane or Kugelrohr vacuum distillation to give 2 $\left(\mathrm{R}^{\mathrm{l}=\mathrm{H}}, \mathrm{R}^{2}=\mathrm{H}, \mathrm{R}^{3}=\mathrm{H}: 80 \%, \mathrm{R}^{\mathrm{l}}=\mathrm{CH}_{3}, \mathrm{R}^{2}=\mathrm{H}, \mathrm{R}^{3}=\mathrm{H}: 78 \%\right.$, $\mathrm{R}^{\mathrm{l}}=\mathrm{H}, \mathrm{R}^{2}=\mathrm{Cl}, \mathrm{R}^{3}=\mathrm{H}: 73 \%, \mathrm{R}^{\mathrm{l}}=\mathrm{H}, \mathrm{R}^{2}=\mathrm{H}, \mathrm{R}^{3}=\mathrm{Br}: 74 \%$ ).

The condensation of 2 was accomplished by the addition of sodium methoxide and benzaldehyes 3 to a solution of 2 in THF at $0^{\circ} \mathrm{C}$. The resulting greenish solution was stirred for $2 \mathrm{~h}$ between $0^{\circ} \mathrm{C}$ and room temperature. After usual aqueous workup. the condensed residue was purified by silica gel chromatography to give $1-(2$-aminophenyl $)-3-$ phenyl-2-propene-l-ones 4 in $76-95 \%$ yields as yellow solids. The condensation proceeded well toward various substituents $\left(\mathrm{CH}_{3}, \mathrm{OCH}_{3}, \mathrm{Cl}\right.$. Br) both on phenyl rings of 2 and 3 . The cyclization of 4 proceeded cleanly by heating with zinc chloride in acetonitrile at $80^{\circ} \mathrm{C}$ for $24 \mathrm{~h}$. The resulting light tan solution was separated by usual acidic workup and the subsequent recrystallization of the residue afforded 2.3-dilydro-2-anyl-4-quinolones 5 in 88-97\% yields as pale yellow solids. The cyclization seems to proceed by the intramolecular conjugate addition of the amino group of 4 to the $\beta$-carbon of the $\alpha \beta$-unsaturated carbonyl group activated by zinc chloride.

The dehy'drogenation of $\mathbf{5}$ was successfully accomplished by heating with (diacetoxyiodo)benzene ${ }^{14}$ under basic condition. A solution of 5 and (diacetoxyiodo)benzene in $0.1 \mathrm{~N}$ methanolic $\mathrm{KOH}$ was heated at $60^{\circ} \mathrm{C}$ for $16 \mathrm{~h}$. The volume of yellow mixture was reduced to a twentieth and the slow addition of $0.05 \mathrm{~N}-\mathrm{HCl}$ resulted in the formation of precipitate. which was filtered and recrystallized in methanol to give 2 -arỵl-4-quinolones 6 in $84-90 \%$ y ields. This dehydrogenation proceeded at $C_{2}$ and $C_{3}$ of hyppervalent iodine intermediate of 5 to give 6 and was found to be general toward various substituents $\left(\mathrm{CH}_{3} . \mathrm{OCH}_{3} . \mathrm{Cl}\right.$. Br) both on the A-ring and B-ring of $\mathbf{5}$. However in the case of methoxy substituted $5(5 \mathrm{c}, 5 \mathrm{~g}$. 5h) a mixture of 6 and 2-aryl-4hydroxyquinolines 7 was obtained. The ratio of keto-enol tautomers was determined by ${ }^{1} \mathrm{H}$ NMR, which showed $\mathrm{C}_{3}$ proton signal of keto form at the $6.12-6.33 \mathrm{ppm}$ and $C_{3}$ proton signal of enol form at the $7.97-8.10 \mathrm{ppm}$. As shown in Table 1. various 2-aryl-4-quinolones were synthesized in overall high yields $(44-64 \%)$ from the starting 2-amino- 
<smiles>[R]c1cc(C(=O)O)c(N)c([R])c1[R]</smiles>
1<smiles>[R]c1cc(C2CC(=O)c3cc([R])c([R])c([R])c3N2[Z])c([Z1])c([R7])c1[R7]</smiles><smiles>[R]c1cc(C(C)=O)c(N)c([R])c1[R]</smiles><smiles>[R]c1cc(C=O)c([R]([H])=O)c([R])c1[R]</smiles><smiles>[R]c1cc(C(=O)/C=C/c2cc([R])c([R])c([R])c2N)c([R])c([R])c1[R]</smiles><smiles>[R]c1cc(-c2cc(O)c3cc([R])c([R])c([R])c3n2)c([R])c([R7])c1[R7]</smiles>

Scheme 1

Table 1. Preparation of compounds 4,5 and 2-ary 1-4-quinolones (6) from 2-aminobenzoic acids

\begin{tabular}{|c|c|c|c|c|c|c|c|c|c|c|}
\hline \multirow{2}{*}{ Entry } & \multirow{2}{*}{$\mathrm{R}^{\mathrm{l}}$} & \multirow{2}{*}{$\mathrm{R}^{2}$} & \multirow{2}{*}{$\mathrm{R}^{3}$} & \multirow{2}{*}{$\mathrm{R}^{4}$} & \multirow{2}{*}{$\mathrm{R}^{4}$} & \multirow{2}{*}{$\mathrm{R}^{6}$} & \multirow{2}{*}{$\mathrm{R}^{?}$} & \multicolumn{3}{|c|}{ Isolated yields, \% } \\
\hline & & & & & & & & 4 & 5 & 6 \\
\hline $\mathbf{a}$ & $\mathrm{H}$ & $\mathrm{H}$ & $\mathrm{H}$ & $\mathrm{H}$ & $\mathrm{H}$ & $\mathrm{H}$ & $\mathrm{H}$ & 94 & 93 & 88 \\
\hline b & $\mathrm{H}$ & $\mathrm{H}$ & $\mathrm{H}$ & $\mathrm{H}$ & $\mathrm{H}$ & $\mathrm{CH}_{3}$ & $\mathrm{H}$ & 89 & 93 & 85 \\
\hline c & $\mathrm{H}$ & $\mathrm{H}$ & $\mathrm{H}$ & $\mathrm{H}$ & $\mathrm{H}$ & $\mathrm{OCH}_{3}$ & $\mathrm{H}$ & 95 & 94 & $90^{\circ}$ \\
\hline d & $\mathrm{H}$ & $\mathrm{H}$ & $\mathrm{H}$ & $\mathrm{H}$ & $\mathrm{H}$ & $\mathrm{Cl}$ & $\mathrm{H}$ & 82 & 90 & 87 \\
\hline $\mathbf{e}$ & $\mathrm{H}$ & $\mathrm{H}$ & $\mathrm{H}$ & $\mathrm{H}$ & $\mathrm{OCH}_{3}$ & $\mathrm{OCH}_{3}$ & $\mathrm{OCH}_{3}$ & 76 & 97 & 89 \\
\hline $\mathrm{f}$ & $\mathrm{CH}_{3}$ & $\mathrm{H}$ & $\mathrm{H}$ & $\mathrm{OCH}_{3}$ & $\mathrm{H}$ & $\mathrm{H}$ & $\mathrm{H}$ & 87 & 91 & 85 \\
\hline g & $\mathrm{H}$ & $\mathrm{Cl}$ & $\mathrm{H}$ & $\mathrm{OCH}_{3}$ & $\mathrm{H}$ & $\mathrm{H}$ & $\mathrm{H}$ & 82 & 88 & $84^{\circ}$ \\
\hline $\mathbf{h}$ & $\mathrm{H}$ & $\mathrm{H}$ & $\mathrm{Br}$ & $\mathrm{H}$ & $\mathrm{H}$ & $\mathrm{OCH}_{3}$ & $\mathrm{H}$ & 84 & 92 & $90^{\circ}$ \\
\hline
\end{tabular}

"A mixture of keto-enol tautomers.

benzoic acids. The reaction worked well with for the methyl (6f). chloro (6g), bromo (6h) substituents on the A-ring and methyl (6b). methoxy (6c. 6e-6g). and chloro (6d) substituents on the B-ring.

\section{Experimental Section}

Preparation of 2'-aminoacetophenone 2a (General procedure). To a solution of 2-aminobenzoic acid ( $823 \mathrm{mg} .6 .0$ mmol) in DME (42 mL) was slowly added methyllithium (1.5 $\mathrm{M}$ in $\mathrm{Et}_{2} \mathrm{O} .13 .2 \mathrm{~mL}, 19.8 \mathrm{mmol}$ ) under argon atmosphere at $0^{\prime \prime} \mathrm{C}$. After being stirred for $\mathrm{l} h$, the resulting tan solution containing white precipitate was quenched with saturated $\mathrm{NH}_{4} \mathrm{Cl}(5 \mathrm{~mL})$ and DME was evaporated in vacto. The mixture was poured into saturated $\mathrm{NH}_{4} \mathrm{Cl}(40 \mathrm{~mL})$. extracted with methylene chloride $(3 \times 25 \mathrm{~mL})$. and washed with saturated $\mathrm{NaHCO}_{3}(40 \mathrm{~mL})$. The combined organic phases were dried over $\mathrm{MgSO}_{4}$. filtered. and concentrated in vacto. The residue was purified by vacuun distillation using Kugelrohr apparatus to give $\mathbf{2 a}(649 \mathrm{mg} .80 \%)$ as a liquid. bp 90-95 ${ }^{\circ} \mathrm{C} / 1.0 \mathrm{~nm} H \mathrm{Hg}:{ }^{1} \mathrm{H}$ NMR $\left(300 \mathrm{MHz}, \mathrm{CDCl}_{3}\right) 87.70$ (dd. $J_{1}=8.3 \mathrm{~Hz}, J_{2}=1.5 \mathrm{~Hz}, \mathrm{IH}$ ). $7.22-7.28(\mathrm{~m}, \mathrm{IH}$ ). 6.61 $6.66(\mathrm{~m}, 2 \mathrm{H}) .6 .28(\mathrm{~s}, 2 \mathrm{H}) .2 .56(\mathrm{~s}, 3 \mathrm{H}):{ }^{13} \mathrm{C} \mathrm{NMR}(75 \mathrm{MHz}$. $\left.\mathrm{CDCl}_{3}\right) \delta 200.7,150.3 .134 .4,132.0 .118 .2 .117 .2,115.7$. 27.8: FT-IR (film) $3467 \& 3341\left(\mathrm{NH}_{2}\right)$. 3072. 2999, I647 (C=O), 1615, 1450. $753 \mathrm{~cm}^{-1}$; MS mz (\%) $135\left(\mathrm{M}^{+} .72\right)$.
$121(8) .120(100), 92(47) .77(3)$.

Preparation of 1-(2'-aminophenyl)-3-phenyl-2-propene1-one ta (General procedure). To a solution of $2 \mathrm{a}$ ( $54 \mathrm{l} \mathrm{mg}$. $4.0 \mathrm{mmol}$ ) in THF (16 mL) was added sodium methoxide (25 wt.\% in $\mathrm{CH}_{3} \mathrm{OH} .1 .0 \mathrm{~mL} .4 .4 \mathrm{mmol}$ ) and benzaldehyde (424 mg. $4.0 \mathrm{~mm}$ ol) at $0^{\circ} \mathrm{C}$. After being stirred for $2 \mathrm{~h}$ between $0^{\circ} \mathrm{C}$ and room temperature. THF was evaporated in vacuo. The mixture was poured into saturated $\mathrm{NH}_{4} \mathrm{Cl}(30$ $\mathrm{mL})$. extracted with methylene chloride $(3 \times 25 \mathrm{~mL})$. and washed with saturated $\mathrm{NaHCO}_{3}(30 \mathrm{~mL})$. The combined organic phases were dried over $\mathrm{MgSO}_{4}$. filtered, and concentrated in vacuo. The residue was purified by silica gel column cluromatography using $30 \% \mathrm{EtOAc} / n$-hexane to give 4a (840 mg. $94 \%)$ as a yellow solid. mp $70-71{ }^{\circ} \mathrm{C}$ (lit. ${ }^{\circ} 71-$ $\left.72{ }^{\circ} \mathrm{C}\right) ;{ }^{1} \mathrm{H} \mathrm{NMR}\left(300 \mathrm{MHz} . \mathrm{CDCl}_{3}\right) \delta 7.86\left(\mathrm{dd}, J_{1}=8.3 \mathrm{~Hz}\right.$, $\left.J_{2}=1.5 \mathrm{~Hz} . \mathrm{lH}\right), 7.74(\mathrm{~d}, J=15.6 \mathrm{~Hz} .1 \mathrm{H}), 7.6 \mathrm{l}(\mathrm{d}, J=15.6$ Hz. lH) $7.60-7.64$ (m, 2H). 7.37-7.43 (m. 3H). 7.25-7.31 (m, 1H), 6.67-6.72 (m. 2H). 6.33 (s. 2H): ${ }^{13} \mathrm{C}$ NMR (75 $\left.\mathrm{MHz}, \mathrm{CDCl}_{3}\right) \delta 191.7,151.0 .142 .9,135.3 .134 .3,131.0$, 130.1, 128.9. 128.2, 123.1. 119.0. 117.3, 115.9. FT-IR (KBr) $3462 \& 3334\left(\mathrm{NH}_{2}\right) .3021,1645(\mathrm{C}=\mathrm{O}), 1614.1575 .1448$. 1212. $1012,746.696 \mathrm{~cm}^{-1}$; MS $m z(\%) 223\left(\mathrm{M}^{+}, 34\right), 222$ (51). $146(100), 120(9) .103(11), 77$ (11).

Preparation of 2,3-dihydro-2-phenyl-4-quinolone 5a (General procedure). A solution of $4 \mathrm{a}(670 \mathrm{mg} .3 .0 \mathrm{mmol})$ and zinc chloride (1.0 M in $\left.\mathrm{Et}_{2} \mathrm{O}, 3.3 \mathrm{~mL}, 3.3 \mathrm{mmol}\right)$ in 
$\mathrm{CH}_{3} \mathrm{CN}(12 \mathrm{~mL})$ was heated to $80^{\circ} \mathrm{C}$ for $24 \mathrm{~h}$. After evaporation of $\mathrm{CH}_{3} \mathrm{CN}$, the light tan mixture was poured into saturated $\mathrm{NH}_{4} \mathrm{Cl}(30 \mathrm{~mL})$ and extracted with methylene chloride $(3 \times 20 \mathrm{~mL})$. The combined organic phases were dried over $\mathrm{MgSO}_{4}$. filtered. and concentrated in vacuo. The residue was recrystallized twice from $10 \% \mathrm{EtOAc} / \mathrm{n}$-hexane to give $\mathbf{5 a}(623 \mathrm{mg} .93 \%)$ as a pale yellow solid. $\mathrm{mp} 150-151$ ${ }^{\circ} \mathrm{C}$ (lit. ${ }^{10} 149-150{ }^{\circ} \mathrm{C}$ ): ${ }^{1} \mathrm{H}$ NMR $\left(300 \mathrm{MHz}, \mathrm{CDCl}_{3}\right) \delta 7.87$ (dd. $\left.J_{1}=7.9 \mathrm{~Hz}, J_{2}=1.5 \mathrm{~Hz}, \mathrm{lH}\right) .7 .32-7.48(\mathrm{~m}, 6 \mathrm{H}) .6 .75-$ $6.81(\mathrm{~m}, \mathrm{lH}), 6.69(\mathrm{~d} . J=8.3 \mathrm{~Hz} . \mathrm{lH}) .4 .76\left(\mathrm{dd}, J_{1}=13.4\right.$ $\left.\mathrm{Hz}, J_{2}=4.1 \mathrm{~Hz} . \mathrm{lH}\right), 4.51(\mathrm{~s}, 1 \mathrm{H}), 2.90\left(\mathrm{dd} . J_{1}=16.2 \mathrm{~Hz} . J_{2}\right.$ $=13.4 \mathrm{~Hz}, 1 \mathrm{H}), 2.78\left(\mathrm{dd}, J_{1}=16.2 \mathrm{~Hz} . J_{2}=4.1 \mathrm{~Hz}, 1 \mathrm{H}\right),{ }^{13} \mathrm{C}$ NMR $\left(75 \mathrm{MHz} . \mathrm{CDCl}_{3}\right) \delta$ 193.7. 151.9. 141.4. 135.8. 129.4. 128.9. 128.0. 127.0. 119.4. 118.9, 116.3. 58.9. 46.9; FT-IR $(\mathrm{KBr}) 3330(\mathrm{~N}-\mathrm{H}), 1655(\mathrm{C}=\mathrm{O}) .1608 .1328 .1154 .761,700$ $\mathrm{cm}^{-1}$; MS $m z(\%) 223\left(\mathrm{M}^{+}, 100\right) .222(44) .146(73), 145$ (15). 119 (19). $77(10)$.

Preparation of 2-phenyl-4-quinolone 6a (General procedure). To a 5 a ( $447 \mathrm{mg} .2 .0 \mathrm{~nm}$ ol) was added a solution of $0.1 \mathrm{~N}-\mathrm{KOH}$ in $\mathrm{CH}_{3} \mathrm{OH}(60 \mathrm{~mL}, 60 \mathrm{mmol})$ and (diacetoxyiodo)benzene ( $709 \mathrm{mg}, 2.2 \mathrm{~nm}$ ol) at room temperature. The mixture was heated to $60^{\circ} \mathrm{C}$ for $16 \mathrm{~h}$. After evaporation of $\mathrm{CH}_{3} \mathrm{OH} .0 .05 \mathrm{~N}-\mathrm{HCl}(50 \mathrm{~mL})$ was slowly added to the mixture at $0{ }^{\circ} \mathrm{C}$. The resulting precipitate was separated by filtration, washed with $\mathrm{H}_{2} \mathrm{O}$, and recrystallized twice in $\mathrm{CH}_{3} \mathrm{OH}$ to give $6 \mathrm{a}(389 \mathrm{mg} .88 \%$ ) as a pale yellow solid. $\mathrm{mp}$ $252-253^{\circ} \mathrm{C}$ (lit ${ }^{\text {tb }} 252-254^{\circ} \mathrm{C}$ ), ${ }^{1} \mathrm{H}$ NMR $(300 \mathrm{MHz}$, DMSOd6) $\delta 11.75(\mathrm{~s}, \mathrm{lH}) .8 .12\left(\mathrm{dd}, J_{1}=8.1 \mathrm{~Hz} . J_{\Sigma}=1.3 \mathrm{~Hz}, 1 \mathrm{H}\right)$. 7.77-7.87 (m. 3H), 7.61-7.71 (m. 1H). 7.57-7.62 (m. 3H). 7.33-7.38 (m. IH). 6.36 (s. IH); ${ }^{13} \mathrm{C}$ NMR (75 MHz. DMSO- $\left.d_{6}\right) \delta 176.8 .150 .0,140.5 .134 .2,131.7 .130 .3,128.9$. 127.3. 124.7, 124.6, 123.2. 118.7. 107.2: FT-IR (KBr) 3260. 3067. 2967, 1635 (C=O), 1582, 1499, 1255.771, $689 \mathrm{~cm}^{-1}$. MS $m z(\%) 22 \mathrm{l}\left(\mathrm{M}^{-}, 100\right), 220(26) .193(63) .165(20), 96$ (8).

2-(4'-Methylphenyl)-t-quinolone (6b). mp 288-290 ${ }^{\circ} \mathrm{C}$ (lit. ${ }^{11} 290-292{ }^{\circ} \mathrm{C}$ ): ${ }^{1} \mathrm{H}$ NMR (300 MHz, DMSO- $\left.d_{6}\right) \delta 11.69$ (s. $1 \mathrm{H}), 8.10$ (dd. $\left.J_{1}=8.1 \mathrm{~Hz}, J_{2}=1.2 \mathrm{~Hz} .1 \mathrm{H}\right), 7.69-7.79$ (m. 3H). 7.63-7.67 (m. 1H). 7.31-7.41 (m. 3H). 6.34 (s. 1H). 2.33 (s. $3 \mathrm{H}){ }^{13} \mathrm{C}$ NMR (75 MHz. DMSO- $\left.d_{6}\right) \delta$ 177.2. 150.4 . 140.7. 140.6. 132.1, 131.6. 129.9. 127.6. 125.1. 125.0. 123.6. 119.1. 107.2. 21.2: FT-IR (KBr) 3263. 3056. 2898. $1633(\mathrm{C}=\mathrm{O}) .1594 .1504,1440,1355,1251,813,753 \mathrm{~cm}^{-1}$. MS $m z(\%) 235\left(\mathrm{M}^{+} .100\right) .234$ (18). $207(59) .206(18), 178$ (9).

2-(4'-Methoxyphenyl)-4-quinolone (6c). $\operatorname{mip} 288-290^{\circ} \mathrm{C}$ : ${ }^{1} \mathrm{H}$ NMR $\left(300 \mathrm{MHz}\right.$. DMSO- $\left.d_{6}\right)$ a mixture of keto-enol tautomer (4:6) $\delta 11.82$ (br s. $1 \mathrm{H}$ ), $8.20 \& 8.09$ (dd. $J_{1}=8.1$ $\left.\mathrm{Hz}, J_{\hat{乏}}=1.2 \mathrm{~Hz} . \mathrm{lH}\right), 8.10$ (s. $\left.0.6 \mathrm{H}\right), 7.81 \& 7.67(\mathrm{~d}, J=8.8$ $\mathrm{Hz}, 2 \mathrm{H}), 7.75-7.79 \& 7.56-7.63(\mathrm{~m}, 2 \mathrm{H}), 7.30-7.36(\mathrm{~m} . \mathrm{lH})$. $7.14 \& 6.96$ (d. $J=8.8 \mathrm{~Hz} .2 \mathrm{H}$ ). 6.33 (s. $0.4 \mathrm{H}$ ). $3.85 \& 3.78$ (s. 3H): FT-IR (KBr) 3216, 3096, 2984, $1634(\mathrm{C}=\mathrm{O}) .1594$. 1505. 1246. 1029. $802.753 \mathrm{~cm}^{-1}$; MS $m z(\%) 25 \mathrm{l}\left(\mathrm{M}^{+}\right.$. 100). $250(28) .236(24) .208(27), 152(10)$.

2-(4'-Chlorophenyl)-4-quinolone (6d). mp $340-342^{\circ} \mathrm{C}$ : ${ }^{1} \mathrm{H}$ NMR $\left(300 \mathrm{MHz}, \mathrm{DMSO}-d_{6}\right) \delta 11.77(\mathrm{~s}, 1 \mathrm{H}), 8.11$ (dd. $J_{1}$ $\left.=8.1 \mathrm{~Hz}, J_{2}=1.2 \mathrm{~Hz}, 1 \mathrm{H}\right) .7 .88(\mathrm{~d} . J=8.6 \mathrm{~Hz}, 2 \mathrm{H}) .7 .76(\mathrm{~d}$.
$J=8.1 \mathrm{~Hz} . \mathrm{lH}), 7.68-7.7 \mathrm{l}(\mathrm{m} . \mathrm{lH}), 7.66(\mathrm{~d} . J=8.6 \mathrm{~Hz} .2 \mathrm{H})$, 7.33.7.38 (m. lH). 6.38 (s, lH): ${ }^{13} \mathrm{C}$ NMR (75 MHz, DMSO$\left.d_{6}\right) \delta 177.3,149.1 .140 .9,135.6,133.4,132.3 .129 .6,129.4$, $128.1,125.0 .123 .8,119.1,107.8$. FT-IR (KBr) 3264. 3059. $2876.1633(\mathrm{C}=\mathrm{O}), 1594.1495 .1251,1092.835,758 \mathrm{~cm}^{-1}$. MS $m z(\%) 257\left(\mathrm{M}^{-}+2,34\right) .255\left(\mathrm{M}^{-}, 100\right), 229(16), 227$ (48). $165(15) .95(10)$.

2-(3', $4^{\prime}, 5^{\prime}$-Trimethoxyphenyl)-4-quinolone (6e). $\mathrm{mp}$ $252.254^{\circ} \mathrm{C}$ (lit. ${ }^{7} 257.258^{\circ} \mathrm{C}$ ): ${ }^{1} \mathrm{H}$ NMR (300 MHz. DMSOd) $\delta 11.62(\mathrm{~s} .1 \mathrm{H}), 8.10$ (dd. $J_{1}=8.1 \mathrm{~Hz}, J_{2}=1.1 \mathrm{~Hz} .1 \mathrm{H}$ ), $7.76($ d. $J=7.8 \mathrm{~Hz} . \mathrm{lH}), 7.64-7.71(\mathrm{~m}, \mathrm{lH}), 7.32 \cdot 7.37(\mathrm{~m}$, lH). $7.11(\mathrm{~s} .2 \mathrm{H}), 6.43(\mathrm{~s}, 1 \mathrm{H}), 3.91(\mathrm{~s}, 6 \mathrm{H}), 3.74(\mathrm{~s}, 3 \mathrm{H}):{ }^{13} \mathrm{C}$ NMR (75 MHz. DMSO- $\left.d_{6}\right) \delta 177.3 .153 .5 .153 .1,150.3$. $140.8,139.5,132.1 .130 .0,125.1 .123 .6,119.0 .107 .6$. 105.4, 60.5. 56.5; FT-IR (KBr) 3262. 2965. $1636(\mathrm{C}=\mathrm{O})$. 1586. $1508,1450,1250.1136,1003,752 \mathrm{~cm}^{-1}$ : MS $m z(\%)$ $311\left(\mathrm{M}^{+} .100\right), 296(25), 268(9) .253(8)$.

8-Methyl-2-(2'-methoxyphenyl)-4-quinolone (6f). $\mathrm{mp}$ $225-226^{\circ} \mathrm{C},{ }^{1} \mathrm{H}$ NMR $\left(300 \mathrm{MHz}, \mathrm{DMSO}-c_{6}\right) \delta 10.65(\mathrm{~s}, \mathrm{lH})$, $7.99($ d. $J=8.0 \mathrm{~Hz} . \mathrm{lH}), 7.49-7.58(\mathrm{~m}, 3 \mathrm{H}), 7.20-7.26(\mathrm{~m}$, $2 \mathrm{H}) .7 .09-7.14(\mathrm{~m}, \mathrm{lH}), 6.16(\mathrm{~s}, \mathrm{lH}) .3 .88(\mathrm{~s} .3 \mathrm{H}), 2.54(\mathrm{~s}$, $3 \mathrm{H}) ;{ }^{13} \mathrm{C}$ NMR $\left(75 \mathrm{MHz}, \mathrm{DMSO}-d_{6}\right) \delta 177.5,157.3,148.7$, $138.9,132.9,131.9 .130 .6 .126 .8,125.3,123.6 .123 .1$. $123.0,121.1,112.4,109.6,56.1$. 17.7 FT-IR (KBr) 3204. $3071.2965,1623(\mathrm{C}=\mathrm{O}), 1552.1456,1241,1026.754 \mathrm{~cm}^{-1}$. MS $m z(\%) 265\left(\mathrm{M}^{-}, 100\right) .250(14) .234$ (40). 233 (19).

7-Chloro-2-(2'-methoxyphenyl)-4-quinolone (6g). $\mathrm{mp}$ 265-267 ${ }^{\circ} \mathrm{C}:{ }^{1} \mathrm{H}$ NMR (300 MHz, DMSO- $d_{6}$ ) a mixture of keto-enol tautomer $(3.7) \delta 11.76$ (br s. $1 \mathrm{H}) .8 .14 \& 8.10$ (d. $J$ $=8.7 \mathrm{~Hz} . \mathrm{lH}), 7.97(\mathrm{~s} .0 .7 \mathrm{H}) .7 .67 \& 7.62(\mathrm{~d}, J=2.0 \mathrm{~Hz}$, lH). $7.48-7.57 \& 7.28-7.36(\mathrm{~m} .3 \mathrm{H}) .7 .23 \& 7.05$ (d. $J=8.1$ Hz. lH). 7.09-7.14 \& 6.94-6.99 (m. lH), 6.12 (s, 0.3H). 3.84 \& 3.71 (s. 3H): FT-IR (KBr) $3210.3069 .2966,1633(\mathrm{C}=0)$. 1598. 1544, 1458, 1238, 1024. 868, $756 \mathrm{~cm}^{-1}$; MS $m z(\%)$ $287\left(\mathrm{M}^{-}+2.34\right), 285\left(\mathrm{M}^{+}, 100\right), 270(25), 256(23) .254(51)$, $179(8)$.

6-Bromo-2-(t'-methoxyphenyl)-t-quinolone (6h). $\mathrm{mp}$ $350-352{ }^{\circ} \mathrm{C}$ (dec.); ${ }^{1} \mathrm{H}$ NMR (300 MHz. DMSO- $d_{6}$ ) a mixture of keto-enol tautomer (3:7) $\delta 12.07$ (br s. $\mathrm{lH}$ ), 8.28 \& $8.16($ d. $J=2.1 \mathrm{~Hz} .1 \mathrm{H}) .8 .17$ (s. $0.7 \mathrm{H}), 7.78-7.83(\mathrm{~m}$. 1H). $7.83 \& 7.67(\mathrm{~d} . J=8.7 \mathrm{~Hz} .2 \mathrm{H}) .7 .57 \& 7.78(\mathrm{~d} . J=9.0$ Hz. 1H). $7.15 \& 6.97$ (d. $J=8.7 \mathrm{~Hz}, 2 \mathrm{H}), 6.39$ (s. $0.3 \mathrm{H}$ ). 3.85 \& 3.78 (s. 3H): FT-IR (KBr) 3260. 3078. 2990, $1634(\mathrm{C}=\mathrm{O})$. 1606. 1489. 1391.1247. 1024. $820 \mathrm{~cm}^{-1}$; MS $m z$ (\%) 331 $\left(\mathrm{M}^{-}+2.97\right), 329\left(\mathrm{M}^{+}, 100\right), 316(19), 314(20) .250(10), 178$ (18).

Acknowledgments. This work was supported by the Korea Research Foundation Grant funded by the Korea Government (MOEHRD). Basic Research Promotion Fund (KRF-2007-005-J13001).

\section{References}

1. (a) Wagmant. A. S.: Wentland. M. P. In Contprehensive Medicinal Chemistry H: Taylor. J. B.: Triggle. D. J.. Eds.: Elsevier: Oxford. U. K.. 2007: Vol. 7. p 567. (b) Ferlin. M. G.; Chiarelotto. G; Gasparotto, V: Via. L. D: Pezzi. V.: Barzon, L.: Palu. G.; 
Castagliuolo. I. J. Afed Chent 2005 . 48.3417.

2. (a) Li. J. J.: Corey. E. J. Nante Reactionts in Heterocktic Chemistry John Wiley \& Sons: New Jersey. U. S. A.. 2005: p 386. (b) Kuo, S. C. Lee, H. Z.; Iuang. I. P.: Lin. Y. T.: Wu, T. S.: Chang. I. J.: Lednicer. D.; Paull. K. D.; Lin, C. M.: Hamel E.: Lee, K. H. J. Med Chem 1993, 36, 1146. (c) Xia. Y.: Yang, Z. Y: Xia. P.: Bastow. K. F.: Nakanishi. Y: Nampoothiri. P.: Hamel. E.: Brossi. A.: Lee. K. H. Bioorg \& Med Chem. Lett. 2003. 13. 2891.

3. Chen. B. C.: Huang. X.: Wang. J. Sinthesis 1987. 482.

4. Mozek: I: Sket B. J. Heterocycl Chem. 1994, $31,1293$.

5. Li. L. Wang. H. K.: Kulo. S. C.: Wu. T. S: Mauger A.: Lin. C. M.: Hamel, E. Lee, K. H. J. Med (Them 1994, 37. 3400 .

6. (a) Hadjeri. M.: Mariotte. A. M.: Boumendjel. A. Chem. Pham. Bull. 2001. 19. 1352. (b) Hadjeri. M: Peiller. E. L: Beney. C: Deka. N.: Lawson. M. A.: Dumontet. C.: Boumendjel. A. J. Afed.
Chent 2004. 47.4964.

7. Li. L.: Wang. H. K.: Kuo. S. C.: Wu. T. S.: Lednicer. D.: Lin1. C. M.: Hamel. E.: Lee. K. H. J. Hed. Chent 1994. 37.1126.

8. (a) Donnelly: J. A.; Farrell. D. F. J. Org. Chem. 1990. 55. 1757. (b) Tokes. A. L.: Litkei. G.; Szilagvi, L. Symth Conmmin. 1992, 22. 2433.

9. Varma. R. S.: Saini. R. K. Synlett 1997,857.

10. Kumar. K. H.: Muralidharan. D.: Perumal. P. T. Sinthesis 2004. 63.

11. Singh. O. V.: Kapil. R. S. Simh. Commm. 1993. 23. 277.

12. Mitsos, C.: Zografos. A.: Igglessi-Markopoulou. O. Chem. Fham. Bull. 2000, 48,211

13. Lee. J. I.; Jung, M. G.; Jung, H. J. Bull. Korean Chem. Soc, 2007. 28.859 .

14. Prakash. O.: Kumar. D.: Saini. R. K.: Singh. S. P. Synth. Conmmm. 1994. 24. 2167. 